\title{
The blue monkey: A new nature inspired metaheuristic optimization algorithm \\ Maha Mahmood $^{1}$ and Belal Al-Khateeb ${ }^{2}$ \\ ${ }^{1}$ College of Computer Science and Information Technology, University of Anbar, Ramadi, Iraq, \\ ${ }^{2}$ College of Computer Science and Information Technology, University of Anbar, Ramadi, Iraq,
}

\section{Article Info}

Mar 02, 2019

\section{Keyword:}

Blue Monkey

Optimization

Metaheuristic

Particle Swarm

Optimization.

\begin{abstract}
This paper introduces a study to Blue Monkey (BM) algorithm, which is a new metaheuristic algorithm optimization based on the performance of blue monkey swarms in nature. The BM algorithm identifies how many males in one group. Normally, outside the season of the breeding, the groups of blue monkeys have only one adult male like other forest guenons. In addition to related patas monkeys (Erythrocebus patas).

Forty-three of well-known test functions, which used in the area of optimization are used as benchmark to check BM algorithm, in addition, $\mathrm{BM}$ verified by a comparative performance check with Artificial-Bee-Colony (ABC), Gravitational Search Algorithm (GSA), Biogeography-Based Optimizer (BBO), and Particle Swarm Optimization (PSO). The obtained results demonstrated that $\mathrm{BM}$ algorithm is competitive compared with the selected metaheuristic algorithms; also, BM is able to converge towards the global optimal through optimization problems. Further, this algorithm is very efficient in field of dissolving real problems with restrictions and unidentified search space. It should be mentioned that the BM algorithm has some variables and it can obtain better results. in many test functions comparing with other algorithms.
\end{abstract}

\section{Corresponding Author:}

Maha Mahmood,

Computer Science,

College of Computer Science and Information Technology, University of Anbar.

Email: maha_mahmood@computer-college.org.

\section{Introduction}

In the past decades, the techniques of meta-heuristic optimization become very common. Moreover, some of these techniques are popular among scientists from various areas and not restricted to the computer scientists. For example, Particle Swarm Optimization (PSO), Genetic Algorithms (GAs) [1], and Ant Colony Optimization (ACO) [2]. Furthermore, beside the big number of theoretical studies, there are many applications to the optimization techniques in different fields. Here, we discuss why meta-heuristics is popular approach [3]. There are four primary reasons can explain this matter: flexibility, simplicity, local optima 
avoidance and derivation-free mechanism. They have been generally based on very simple concepts [4]. Usually, there is a relationship between the inspiration and physical phenomena, the behavior of animals, or evolutionary concepts. Several natural concepts can be simulated by computers. The simplicity makes this possible, in addition to suggest new metaheuristics, hybridize two or more metaheuristics, or enhancing the current meta-heuristics. Furthermore, the simplicity makes the process of learning meta-heuristics fast and easy to the other scientists and can be applied to their problems. The PSO seeking the inputs to find the best ones, which have the best fitness value over the course of iteration in order to enhance the rest of solutions. Because of gradient-free approach, low probability of local optima stagnation, and high flexibility [5], there are many meta-heuristics applications used in various science and industry areas [6]. Unlike single- objective optimization, it's not possible to have one solution in case of several objectives as the goal of the optimization process. In this instance, the asset of solution which state various trade-offs between the aims, involves optimal solutions of a multi-objective problem [7]. EAs is characterized by many benefits in contrast to conventional optimization techniques, like derivative-free approach and flexibility. Those two benefits are due to the stochastic nature of EAs that helps them in case of an optimization problem like a black box and optimizing it without gradient information about its search space. Because of the extraction of gradient information of real problems is not trifling or sometimes not possible [8]. The techniques of derivative free enhancement are of higher relevance. Furthermore, random operators can stop EAs from easily stagnating in local optima in comparison with traditional gradient-based optimization approaches [9]. There are many suggested algorithms in this field, but the big question is that is there a need for more optimization approaches. The answer is related to a theorem called No Free Lunch (NFL) [10]. Based on this theorem, there is no one can suggest an algorithm to solve all the optimization related problems. So, if an algorithm succeeds to solve a particular set of tasks, doesn't mean it can solve the rest problems which have different type and nature. Furthermore, this is the motivation of this study, where a simple yet efficient optimization technique is suggested in order to deal with real optimization problem with not known search spaces [11]. This work introduces a study about designing optimization algorithms using a simple mathematical function in the meta-heuristic field.

\section{Literature review}

In case of optimization with single-objective, one solution is available only which is the global optimum. This is due to the unary objective in the problems with single-objective, and the presence of only single best solution. The process of comparing solutions becomes easy in case of existing one objective and is done by the relational operators: $>, \geq,<, \leq$, or $=$. The nature of these problems lies on optimization problems and comparing the nominated solutions then identify the best solution. In case of the problems with multiobjective, the comparison of solutions must be with more than one objective (criterion) [12]. To find a solution to a multi-objective problem, we must use a set of solutions which is known as the Pareto optimal set, representing the best tradeoffs between objectives. In the past years, a considerable multi-objective algorithms were introduced. There are some of them which are well-known and have stochastic population-based: Strength Pareto Evolutionary Algorithm (SPEA) [8], Non-dominated sorting Genetic Algorithm version2 (NSGA-II), Non dominated Sorting Genetic Algorithm [7], Multi-Objective Evolutionary Algorithm based on Decomposition (MOEA/D), Multi Objective Particle Swarm Optimization (MOPSO) [8], Pareto-frontier Differential Evolution (PDE), and Pareto Archived Evolution Strategy (PAES). Two primary ingredient when enhancing or optimizing an EA are test problems and performance measures [13]. Test problem used usually to benchmark the abilities of EAs, the efficiency of EAs is determined by performance metrics from different perspectives. Moreover, the designer used performance metrics for conducting quantitative comparative researches. The studies said that there is a significant number of performance measures in the area of evolutionary multi-objective optimization (EMOO) [14]. There are different performance metrics used for comparing algorithms in the area of EMOO. This is because the complication of the process of optimization and multi-objectivity, and the existence of several performance metrics gives an objective and fair comparison. The various sections of EMOO require certain or adapted measures for the quantification of the efficiency of algorithms in an effective manner as the literatures said [15], In spite of its big important, no performance measure in the area of robust multi-objective optimization (RMOO). Due to this, we suggested 3 new measures of performance for robust multi-objective algorithms. There are several reasons make them not working well in many cases, like the complicated nature of optimization tasks, constant and linear timevarying values for social and cognitive factors. The performance can be improved -in some cases- when 
utilizing a nonlinear time-varying coefficient for PSO. Furthermore, using only one time-varying nonlinear strategy for all particles cannot cause a general sufficient optimizer [14].

\section{The blue monkey}

In this section, the motivation of the suggested approach and the mathematical model are discussed.

\subsection{The inspiration of the blue monkeys}

Cercopithecus mitis joins with Cercopithecus ascanius (red tailed monkey) for additional protection. "Cercopithecus mitis social system is mainly female because the males leave once, they are mature". The male Cercopithecus mitis have little or no interaction with the young. Cercopithecus mitis are quite regional, for this reason the young males should get out rapidly in order to help themselves to be more successful [15]. They challenge the dominant male of some other family. In the case where they defeat it, they dominate the leading of that family, this behavior will provide food, a place to live and socialize for young males [15]. Cercopithecus mitis are referred to as nomadic. The blue monkeys have a propensity to waste time in the forest habitats, this related to existence of the fruits resource and structural characteristics like larger fruit patches [16].

The blue monkeys are not like the other kinds of monkeys. Normally they live in female dominated social systems, which mean that the females are staying in their natal groups. On the other hand, the males leave their groups as soon as reaching the stage of maturity [17]. Most blue monkey groups having a lot of females and babies, but only one male. This matter makes inbreeding difficult. The males leave the group to another group when they reach maturity, but finding another group may take some time, so they may seem as solitary males. In the field of social relationships, the blue monkeys don't have very powerful instincts [18]. The time of social interactions is short, this happened normally during playing together and grooming one another. Also, there is an interaction between babies and their mothers and other adults in the group. This lead to those babies doesn't usually go near their male counterparts. All operating is really handlers of the babies. The young females look after babies, carry them and keep them safe. This habit teaches babies to react with all monkeys in later life [19].

\subsection{Mathematical model and algorithm}

The algorithm of BM together with its mathematical model are presented in this section.

\subsubsection{Group division}

The BM algorithmic program mimics behavior of the Blue Monkey. To model such interactions, every cluster of monkey' area unit needed to maneuver over the search area. As mentioned earlier, the Monkeys when being divided into teams who begin to look for places of food at long distances area and stronger monkey not among the scope of traditional vision. The male Cercopithecus mitis have little to no interaction with the young ones. Because of the territorial nature of the cercopithecus mitis, the young males should go out as fast as possible in order to become more successful. They will enter a challange with the dominant male of another family. In the case where they success to defeat that male, they can be the leaders of this family, so they can offer food supplies, place to live and socialization for young males [20]. Normally, the groups of blue monkeys having one male and a big number of femals and babies [20].

\subsubsection{Update position}

The update position for each blue monkey in the group depends on the best blue monkey position in that group, this behavior is delineating by the following equations: 
Rate $_{i+1}=\left(0.7 *\right.$ Rate $\left._{i}\right)+\left(\mathbf{W}_{\text {leader }}-\mathbf{W}_{\mathrm{i}}\right) *$ rand $^{*}\left(\mathbf{X}_{\text {best }}-\mathbf{X}_{\mathrm{i}}\right)$

$\mathbf{X}_{\mathrm{i}+1=} \mathbf{X}_{\mathrm{i}}+$ Rate $_{\mathrm{i}+1 * \text { rand }}$

where Rate represents the monkey power rate, Wleader is the leader weight, $\mathrm{Wi}$ is the monkey weight at which all weights are random numbers between [4,6], $\mathrm{X}$ is the monkey position, Xbest is the leader position and rand is an arbitrary number between $[0,1]$.

Also, in order to update the children of blue monkey, the following equations are used:

$\operatorname{Rate}^{\text {ch }}{ }_{(i+1)}=\left(0.7 * \operatorname{Rate}^{\text {ch }}{ }_{i}\right)+\left(W^{\text {ch }}{ }_{\text {leader }}-W^{\text {ch }}{ }_{i} * \operatorname{rand}^{*}\left(\mathbf{X}_{\text {best }}^{\text {ch }}-X^{\text {ch }}{ }_{i}\right)\right.$

$\mathbf{X}_{(i+1)}^{\mathrm{ch}}=\mathbf{X}_{\mathrm{i}}^{\mathrm{ch}}+\operatorname{Rate}_{(\mathrm{i}+1)}^{\mathrm{ch}} *$ rand

where Ratech represents the child power rate, Wchleader is the leader child weight, Wchi is the child weight at which all weights are random numbers between $[4,6], \mathrm{Xch}$ is the child position, Xchbest is the leader child position and "rand" representing an arbitrary number between $[0,1]$. The position should be updated in each iteration.

\section{Algorithm1: BM optimization}

1- Initialize the blue monkey and children population bi ( $\mathrm{i}=1 \ldots \mathrm{n})$.

2- Initialize Power Rate Rate and Weight $\boldsymbol{W}$. where (Rate $\in[0,1]),(\mathrm{W} \in[4,6])$

3- Distribute the blue monkeys randomly into teams (T), while all children in one team.

4- Calculate the fitness of children and all blue monkeys in each group.

5- For each group, select the worst value and the best value of fitness and store it in Current Best. While children select the best fitness.

6- $\mathrm{t}=1$.

7- While ( $\mathrm{t} \leq$ maximum number of iterations)

8. Swapping the worst fitness in each group by the best fitness in children group.

9- Update Rate and $\mathbf{X}$ position of all blue monkeys in each group by Equations 1 and 2.

10- Update Rate and $\mathbf{X}$ position of children by Equations 3 and 4.

11- Update the fitness of all blue monkey and children.

12- Update Current Best:

if New Best is better than Current Best Then Current Best=New Best.

13- $\mathrm{t}=\mathrm{t}+1$.

14- End While.

15- Return the optimal blue monkey.

\section{Results and discussion}

In this section, BM algorithm is tested and evaluated by using 43 benchmark functions. Some of those functions (the first 18) are simple and traditional functions that are used by many researchers (Storn and Price 1997). Those functions are chosen for being able to compare our results with the results of some well-known metaheuristic algorithms. The selected 43 test functions are shown in table 1 and table 2 where $\mathrm{D}$ means the dimension of the function, Range is the search space limits of the function and Opt is the optimal value.

In general, the utilized test functions are minimization functions that are either unimodal or multimodal benchmark functions.

The suggested BM algorithm was performed 30 times for every one of the benchmark functions. The statistical results (average and standard deviation) are presented in tables 3 and 4. For proving the results, BM algorithm is compared to ABC [12], PSO [21], GSA [22], GWO and BBO [23]. 


\subsection{Exploitation analysis}

The results in table 3 show that BM outperforms the other selected algorithm in most of the in most of the selected 18 functions for the unimodal functions.

The unimodal functions are appropriate for benchmarking exploitation according to the formally mentioned operators. Which is why, the obtained results demonstrate the superiority of BM in exploiting the optimal value; Figure 1 depicts the comparison of average value taken between BM, GWO, BBO, PSO and ABC for unimodal benchmark functions.

\subsection{Exploration analysis}

Many local optima can be found in multimodal functions with the number growing exponentially with dimension. Therefore, the multimodal functions are appropriate for testing the exploration strength of an algorithm. The results in table 4 demonstrated that BM outperforms ABC, GSA, PSO and GWO in most of the selected 25 functions for the multimodal functions.

$\mathrm{BM}$ is able to be of a comparable performance with $\mathrm{BBO}$; and outperforms it in sometimes. The obtained results demonstrate that the $\mathrm{BM}$ algorithm superiority in terms of exploration; Figure 2 depicts the comparison of average value taken between $\mathrm{BM}, \mathrm{GWO}, \mathrm{BBO}, \mathrm{PSO}$ and $\mathrm{ABC}$ for multimodal benchmark functions. The Unimodal Benchmark Functions has been tested in many studies [23-27].

Table1. Unimodal benchmark functions

\begin{tabular}{|c|c|c|c|c|}
\hline Equation & $\begin{array}{l}\text { Test } \\
\text { Name }\end{array}$ & D & Range & Opt \\
\hline$f_{1}(x)=\sum_{i=1}^{n} x_{i}^{2}$ & Sphere & 30 & $100,-100$ & 0 \\
\hline$f_{2}(x)=\sum_{i=1}^{n}\left|x_{i}\right|+\prod_{i=1}^{n}\left|x_{i}\right|$ & $\begin{array}{c}\text { Schwefel } \\
2.22\end{array}$ & 2 & $100,-100$ & 0 \\
\hline$f_{3}(x)=\max _{i}\left\{\left|x_{i}\right|, 1 \leq i \leq n\right\}$ & $\begin{array}{c}\text { Schwefel } \\
2.21\end{array}$ & 2 & $100,-100$ & 0 \\
\hline$f_{5}(x)=\sum_{i=1}^{n}\left(\sum_{i=1}^{i} x_{j}\right)^{2}$ & Schwefel 1.2 & 2 & $100,-100$ & 0 \\
\hline$f_{6}(x, y)=-200 e \sqrt[-0.2]{x^{2}+y^{2}}$ & Ackley 2 & 2 & $32,-32$ & -200 \\
\hline $\begin{array}{c}f_{7}(x)=x_{1}{ }^{2}+x_{2}{ }^{2}-0.3 \cos \cos \left(3 \pi x_{1}\right)-0.4 \cos \cos \left(4 \pi x_{2}\right) \\
+0.7\end{array}$ & $\begin{array}{c}\text { Bohachevsky } \\
\text { n N.1 }\end{array}$ & 2 & $100,-100$ & 0 \\
\hline$f_{8}(x)=\left(x_{1}+2 x_{2}-7\right)^{2}+\left(2 x_{1}+x^{2}-5\right)^{2}$ & Booth & 2 & $10,-10$ & 0 \\
\hline$f_{9}(x)=\sum_{i=1}^{n} x_{i}^{2}+\left(\sum_{i=1}^{n} 0.5 i x_{i}\right)^{2}+\left(\sum_{i=1}^{n} 0.5 i x_{i}\right)^{4}$ & Zakharov & 2 & $5.12,-5.12$ & 0 \\
\hline$f_{10}(x)=\left(x_{1}-1\right)^{2}+\sum_{i=2}^{d} \quad i\left(2 x_{i}^{2}-x_{i-1}\right)^{2}$ & Dixon-Price & 2 & $10,-10$ & 0 \\
\hline$f_{11}(x)=-\exp \left(-0.5 \sum_{i=1}^{n} \quad x_{i}^{2}\right.$ & Exponential & 30 & $1,-1$ & -1 \\
\hline$f_{12}(x)=0.26\left(x_{1}{ }^{2}+x_{2}{ }^{2}\right)-0.48 x_{1} x_{2}$ & Matyas & 2 & $10,-10$ & 0 \\
\hline$f_{13}(x)=\sum_{i=1}^{n} \quad x_{i}^{10}$ & $\begin{array}{c}\text { Schwefel } \\
2.23\end{array}$ & 2 & $100,-100$ & 0 \\
\hline$f_{14}(x)=\sum_{i=1}^{n} \quad\left|x_{i}\right|$ & $\begin{array}{c}\text { Schwefel } \\
2.20\end{array}$ & 2 & $100,-100$ & 0 \\
\hline$f_{15}(x)=0.5+\frac{\sin ^{2}\left(x^{2}-y^{2}\right)-0.5}{\left(1+0.001\left(x^{2}+y^{2}\right)\right)^{2}}$ & Schaffer N2 & 2 & $36,-36$ & 0 \\
\hline
\end{tabular}


PEN Vol. 7, No. 3, September 2019, pp.1054-1066

\begin{tabular}{|c|c|c|c|c|}
\hline$f_{16}(x)=0.5+\frac{\sin ^{2}\left(\cos \cos \left(\left|x^{2}-y^{2}\right|\right)\right)-0.5}{\left(1+0.001\left(x^{2}+y^{2}\right)\right)^{2}}$ & Schaffer N3 & 2 & $100,-100$ & $\begin{array}{c}0.0015 \\
6\end{array}$ \\
\hline$f_{17}(x)=0.5+\frac{\sin ^{2}\left(x_{1}^{2}-x_{2}^{2}\right)^{2}-0.5}{\left[1+0.001\left(x_{1}^{2}-x_{2}^{2}\right)\right]^{2}}$ & Schaffer N1 & 2 & $100,-100$ & 0 \\
\hline$f_{18}(x)=\sum_{i=1}^{\frac{d}{4}} \quad \begin{array}{l}\left(x_{4 i-3}+10 x_{4 i-2}\right)^{2}+5\left(x_{4 i-1}+x_{4 i}\right)^{2} \\
\left.+\left(x_{4 i-2}+x_{4 i-1}\right)^{4}+10\left(x_{4 i-3}+x_{4 i}\right)^{4}\right]\end{array}$ & Powell & 10 & $5,-4$ & 0 \\
\hline$f_{19}(x)=\sum_{i=1}^{d} \quad\left[\begin{array}{ll}\left(\sum_{j=1}^{d}\right. & \left.x_{j}^{i}\right)-b_{i}\end{array}\right]^{2}$ & Power Sum & 4 & 4,0 & 0 \\
\hline
\end{tabular}

Table 2. Multi-modal benchmark functions

\begin{tabular}{|c|c|c|c|c|c|}
\hline Equation & Test Name & Typ & D & Range & Opt \\
\hline $\begin{array}{r}f_{20}(x)=-20 \exp \exp \left(\sum_{i=1}^{-0.2} x_{i}^{2}\right)- \\
\quad \exp \exp \left(\frac{1}{n} \sum_{i=1}^{n} \cos \cos \left(2 \pi x_{i}\right)\right)+20+e\end{array}$ & Ackley & $\mathrm{N}$ & 2 & $10,-10$ & 0 \\
\hline$f_{21}(x)=\sum_{i=1}^{n}\left\lfloor\left|x_{i}\right|\right\rfloor$ & Step 1 & $\mathrm{~N}$ & 30 & $100,-100$ & 0 \\
\hline$f_{22}(x)=\sum_{i=1}^{n} i x_{i}^{4}+\operatorname{random}[0,1)$ & Quartic & $\mathrm{N}$ & 10 & $1.28,-1.28$ & 0 \\
\hline$f_{23}(x)=\left(4-2.1 x_{1}^{2}+\frac{x_{1}^{4}}{3}\right) x_{1}^{2}+x_{1} x_{2}+\left(-4+4 x_{2}^{2}\right) x_{2}^{2}$ & $\begin{array}{l}\text { Six-Hump } \\
\text { Camel }\end{array}$ & $\mathrm{N}$ & 2 & $5,-5$ & -1.0316 \\
\hline$f_{24}(x)=a\left(x_{2}-b x_{1}^{2}+c x_{1}-r\right)^{2}+s(1-t) \cos \left(x_{1}\right)+s$ & Branin & & 2 & $15,-5$ & 0.3979 \\
\hline $\begin{array}{c}f_{25}(x)=\left[1+\left(x_{1}+x_{2}+1\right)^{2}\left(19-14 x_{1}+3 x_{1}^{2}-14 x_{2}+6 x_{1} x_{2}+3 x_{2}^{2}\right)\right] *\left[30+\left(2 x_{1}\right.\right. \\
\left.\left.+3 x_{2}\right)^{2}\left(18-32 x_{1}+12 x_{1}^{2}-48 x_{2}+36 x_{1} x_{2}+27 x_{2}^{2}\right)\right]\end{array}$ & $\begin{array}{l}\text { Goldstein } \\
\text { Price }\end{array}$ & $\mathrm{N}$ & 2 & $2,-2$ & 3 \\
\hline$f_{26}(x)=-\sum_{i=1}^{4} c_{i} \exp \left(-\sum_{j=1}^{3} a_{i j}\left(x_{j}-p_{i j}\right)^{2}\right)$ & $\begin{array}{l}\text { Hartmann } \\
\text { 3-D }\end{array}$ & $\mathrm{F}$ & 3 & 1,0 & -3.8628 \\
\hline$f_{27}(x)=-\sum_{i=1}^{4} c_{i} \exp \left(-\sum_{j=1}^{6} a_{i j}\left(x_{j}-p_{i j}\right)^{2}\right)$ & $\begin{array}{l}\text { Hartmann } \\
\quad 6-D\end{array}$ & $\mathrm{~F}$ & 6 & 1,0 & -3.3224 \\
\hline$f_{28}(x)=418.9829 n-\sum_{i=1}^{n} \quad x_{i} \sin \left(\sqrt{\left|x_{i}\right|}\right)$ & $\begin{array}{l}\text { Schwefel } \\
2.26\end{array}$ & $\mathrm{~N}$ & 30 & $500,-500$ & -12569.5 \\
\hline $\begin{array}{c}f_{29}(x)=\frac{\pi}{30}\left\{10 \sin ^{2}\left(\pi y_{1}\right)+\sum_{i=1}^{n-1}\right. \\
\left.\left(y_{n}-1\right)^{2}\right\}+\sum_{i=1}^{n} \quad u\left(x_{i}, 10,100,4\right)\end{array}$ & $\begin{array}{c}\text { Penalized } \\
1\end{array}$ & $\mathrm{~N}$ & 2 & $50,-50$ & 0 \\
\hline $\begin{array}{c}f_{30}(x)=0.1\left\{\sin ^{2}\left(3 \pi y_{1}\right)+\sum_{i=1}^{n-1} \quad\left(x_{i}-1\right)^{2}\left[1+\sin ^{2}\left(3 \pi y_{i+1}\right)\right]+\right. \\
\left.\left(x_{n}-1\right)^{2}\right\}+\sum_{i=1}^{n} \\
u\left(x_{i}, 5,100,4\right)\end{array}$ & $\begin{array}{l}\text { Penalized } \\
2\end{array}$ & $\mathrm{~N}$ & 2 & $50,-50$ & 0 \\
\hline$f_{31}(x)=2 x_{1}^{2}-1.05 x_{1}^{4}+\frac{x_{1}^{6}}{6}+x_{1} x_{2}+x_{2}^{2}$ & $\begin{array}{l}\text { Three- } \\
\text { Hump } \\
\text { Camel }\end{array}$ & $\mathrm{N}$ & 2 & $5,-5$ & 0 \\
\hline$f_{32}(x)=-200 e^{-0.2 \sqrt{x^{2}+y^{2}}}+5 e^{\cos \cos (3 x)+\sin \sin (3 y)}$ & Ackley 3 & & 2 & $32,-32$ & -195.629 \\
\hline$f_{33}(x)=x_{1}^{2}+2 x_{2}^{2}-0.3 \cos \cos \left(3 \pi x_{1}\right) \cos \cos \left(4 \pi x_{2}\right)+0.3$ & $\begin{array}{l}\text { Bohachevs } \\
\text { kyn N.2 }\end{array}$ & $\mathrm{N}$ & 2 & $10,-10$ & 0 \\
\hline $\begin{array}{c}f_{34}(x)=\sin \sin (x) e^{(1-\cos \cos (y))^{2}}+\cos \cos (x) e^{(1-\sin \sin (x))^{2}}+ \\
(x-y)^{2}\end{array}$ & Brid & $\mathrm{N}$ & 2 & $2 p i,-2 p i$ & $\begin{array}{c}- \\
106.764 \\
5\end{array}$ \\
\hline
\end{tabular}


PEN Vol. 7, No. 3, September 2019, pp.1054-1066

\begin{tabular}{|c|c|c|c|c|c|}
\hline $\begin{array}{c}f_{35}(x)=\left(\left|\sin \sin \left(x_{1}\right) \sin \sin \left(x_{2}\right) \exp \left(\left|100-\frac{\sqrt{x_{1}^{2}+x_{2}^{2}}}{\pi}\right|\right)\right|\right. \\
+1)^{0.1}\end{array}$ & $\begin{array}{c}\text { Cross in } \\
\text { Tiny }\end{array}$ & $\mathrm{N}$ & 2 & $10,-10$ & -2.06261 \\
\hline$f_{36}(x)=-\cos \cos \left(x_{1}\right) \cos \left(x_{2}\right) \exp \left(-\left(x_{1}-\pi\right)^{2}-\left(x_{2}-\pi\right)^{2}\right)$ & Easom & $\mathrm{F}$ & 2 & $100,-100$ & -1 \\
\hline$f_{37}(x)=-\frac{\sin ^{2}(x-y) \sin ^{2}(x+y)}{\sqrt{\left(x^{2}+y^{2}\right)}}$ & Keane & $\mathrm{N}$ & 2 & 10,0 & -0.6737 \\
\hline$f_{38}(x)=-\sum_{i=1}^{d}\left(x_{i}-1\right)^{2}-\sum_{i=2}^{d} x_{i} x_{i-1}$ & Trid & $\mathrm{F}$ & 6 & 36,36 & -50 \\
\hline$f_{39}(x)=-\left(x_{2}+47\right) \sin \left(\sqrt{\left.\left|x_{2}+\frac{x_{1}}{2}+47\right|\right)}-x_{1} \sin \left(\sqrt{\mid x_{1}-\left(x_{2}+47 \mid\right.}\right)\right.$ & Egg Holder & $\mathrm{N}$ & 2 & $512,-512$ & -959.641 \\
\hline$f_{40}(x)=\frac{\operatorname{sinsin}(10 \pi x)}{2 x}+(x-1)^{4}$ & $\begin{array}{l}\text { Gramacy \& } \\
\text { Lee }\end{array}$ & $\mathrm{F}$ & 1 & $2.5,0.5$ & -0.869 \\
\hline$f_{41}(x)=-\left|\sin \sin \left(x_{1}\right) \exp \left(\left|1-\frac{\sqrt{x_{1}^{2}+x_{2}^{2}}}{\pi}\right|\right)\right|$ & Holder & $\mathrm{N}$ & 2 & $10,-10$ & -19.2085 \\
\hline$f_{42}(x)=418.9829 d-\sum_{i=1}^{n} \quad x_{i} \sin \left(\sqrt{\left|x_{i}\right|}\right)$ & Schwefel & $\mathrm{N}$ & 2 & $500,-500$ & 0 \\
\hline$f_{43}(x)=-\sum_{i=1}^{d} \quad \sin \sin \left(x_{i}\right) \sin ^{2 m}\left(\frac{i_{i}^{2}}{\pi}\right)$ & $\begin{array}{l}\text { Michalewi } \\
\quad \text { cs }\end{array}$ & $\mathrm{N}$ & 2 & $2.21,1.57$ & -1.8013 \\
\hline$f_{44}(x)=\sum_{i=1}^{d} \quad \frac{x_{i}^{2}}{4000}-\prod_{i=1}^{d} \quad \cos \cos \left(\frac{x_{i}}{\sqrt{i}}\right)+1$ & Griewank & $\mathrm{N}$ & 30 & $600,-600$ & 0 \\
\hline
\end{tabular}

Table 3. Results of unimodal benchmark functions

\begin{tabular}{|c|c|c|c|c|c|c|}
\hline \multirow[t]{2}{*}{ Function } & \multicolumn{2}{|c|}{ BM } & \multicolumn{2}{|c|}{$A B C$} & \multicolumn{2}{|c|}{ PSO } \\
\hline & Average & Std & Average & Std & Average & Std \\
\hline F1 & $9.03693 \mathrm{E}-11$ & $1.78213 \mathrm{E}-10$ & $1.25 \mathrm{E}-03$ & $8.11 \mathrm{E}-04$ & $2.54 \mathrm{E}-08$ & $7.98 \mathrm{E}-08$ \\
\hline F2 & $3.61157 E-47$ & $9.04387 \mathrm{E}-47$ & $1.66 \mathrm{E}-03$ & $1.10 \mathrm{E}-03$ & $2.03 E-51$ & $1.10 \mathrm{E}-50$ \\
\hline F3 & 33.67989 & $\begin{array}{c}20.2171660 \\
2\end{array}$ & $1.71 \mathrm{E}+02$ & $8.05 E+01$ & 75.79913 & 99.69846 \\
\hline F4 & $1.10936 \mathrm{E}-45$ & 3.30122E-45 & $5.62 \mathrm{E}-04$ & $2.78 \mathrm{E}-04$ & $9.14 \mathrm{E}-46$ & $5.01 E-45$ \\
\hline F5 & $8.8817 \mathrm{E}-16$ & $2.97462 E-31$ & $3.46 \mathrm{E}-04$ & $1.78 \mathrm{E}-04$ & $1.95 \mathrm{E}-15$ & $1.66 \mathrm{E}-15$ \\
\hline F6 & $4.20319 E-10$ & $6.61705 \mathrm{E}-10$ & $5.42 \mathrm{E}-01$ & 4.57E-01 & $1.19 \mathrm{E}-08$ & $1.94 \mathrm{E}-08$ \\
\hline F7 & -1.0316 & $2.22045 \mathrm{E}-16$ & -1.0316 & $6.78 \mathrm{E}-16$ & $1.58 \mathrm{E}+01$ & $9.15 \mathrm{E}+01$ \\
\hline F8 & $2.3558 \mathrm{E}-31$ & 0 & $1.75 \mathrm{E}-07$ & $1.67 E-07$ & $2.36 \mathrm{E}-31$ & $4.45 E-47$ \\
\hline F9 & $1.3498 \mathrm{E}-32$ & $2.73691 E-48$ & $5.12 \mathrm{E}-07$ & $6.48 \mathrm{E}-07$ & $1.35 E-32$ & $5.57 \mathrm{E}-48$ \\
\hline F10 & -195.629 & $5.75496 \mathrm{E}-14$ & $-1.96 E+02$ & $5.78 \mathrm{E}-14$ & $-1.96 E+02$ & 3.57E-05 \\
\hline F11 & -2.0626 & 0 & -2.0626 & $1.36 \mathrm{E}-15$ & -2.06234 & $1.50 \mathrm{E}-03$ \\
\hline F12 & -1 & 0 & -1 & 0 & -1 & 0 \\
\hline F13 & -0.6737 & $1.11022 \mathrm{E}-16$ & -0.67367 & 0 & -0.6737 & $1.13 \mathrm{E}-16$ \\
\hline
\end{tabular}


PEN Vol. 7, No. 3, September 2019, pp.1054-1066

\begin{tabular}{|c|c|c|c|c|c|c|}
\hline $\mathbf{F 1 4}$ & -50 & 0 & -49.9974 & $7.23 \mathrm{E}-14$ & -50 & 0 \\
\hline $\mathbf{F 1 5}$ & -0.869 & $1.16441 \mathrm{E}-16$ & -2.8739 & $9.03 \mathrm{E}-16$ & -0.625 & 0 \\
\hline $\mathbf{F 1 6}$ & -19.2085 & $3.55271 \mathrm{E}-15$ & N/A & N/A & -15.1402 & $5.42 \mathrm{E}-15$ \\
\hline $\mathbf{F 1 7}$ & -1.8013 & 0 & -1.878847 & $7.84 \mathrm{E}-02$ & -1.80109 & $3.60 \mathrm{E}-04$ \\
\hline $\mathbf{F 1 8}$ & $\begin{array}{c}0.00499278 \\
8\end{array}$ & $\begin{array}{c}0.00416523 \\
7\end{array}$ & 0.8444203 & 0.1298731 & $6.81 \mathrm{E}-03$ & $8.86 \mathrm{E}-03$ \\
\hline
\end{tabular}

Table 4. Results of unimodal benchmark functions

\begin{tabular}{|c|c|c|c|c|c|c|}
\hline \multirow{2}{*}{$\begin{array}{l}\text { Functi } \\
\text { on }\end{array}$} & \multicolumn{2}{|c|}{ BBO } & \multicolumn{2}{|c|}{ GSA } & \multicolumn{2}{|c|}{ GWO } \\
\hline & Average & Std & Average & Std & Average & Std \\
\hline F1 & 2.77E-03 & $6.89 \mathrm{E}-04$ & $2.14 \mathrm{E}-17$ & $5.64 \mathrm{E}-18$ & $8.32 \mathrm{E}-62$ & $2.01 \mathrm{E}-61$ \\
\hline F2 & $4.46 \mathrm{E}-07$ & $6.06 \mathrm{E}-07$ & $6.02 \mathrm{E}-11$ & 2.99E-11 & $1.26 \mathrm{E}-189$ & 0 \\
\hline F3 & $2.89 E+01$ & $1.43 \mathrm{E}+01$ & $2.61 \mathrm{E}+01$ & $1.78 \mathrm{E}-01$ & $2.67 E+01$ & 7.04E-01 \\
\hline F4 & $1.70 \mathrm{E}-08$ & $3.53 \mathrm{E}-08$ & $1.52 \mathrm{E}-10$ & $6.47 \mathrm{E}-11$ & $3.21 \mathrm{E}-214$ & 0 \\
\hline F5 & 4.74E-09 & $1.64 \mathrm{E}-08$ & $1.71 \mathrm{E}-10$ & 1.07E-10 & $8.88 \mathrm{E}-01$ & $4.01 \mathrm{E}-31$ \\
\hline F6 & 1.06361 & 2.07E-01 & $2.23 \mathrm{E}-17$ & $5.39 \mathrm{E}-18$ & $5.52 \mathrm{E}-01$ & $3.23 \mathrm{E}-01$ \\
\hline F7 & -1.0316 & $6.78 \mathrm{E}-16$ & -1.0316 & $6.78 \mathrm{E}-16$ & -1.03162 & 4.31E-09 \\
\hline F8 & $1.91 \mathrm{E}-10$ & $4.11 \mathrm{E}-10$ & $3.16 \mathrm{E}-21$ & $2.91 \mathrm{E}-21$ & $1.45 \mathrm{E}-08$ & $1.50 \mathrm{E}-08$ \\
\hline F9 & $3.41 \mathrm{E}-12$ & $1.70 \mathrm{E}-11$ & $1.84 \mathrm{E}-21$ & $1.57 E-21$ & $1.48 \mathrm{E}-08$ & $1.90 \mathrm{E}-08$ \\
\hline F10 & $-1.96 E+02$ & $5.78 \mathrm{E}-14$ & -195.629 & $5.78 \mathrm{E}-14$ & -195.629 & $2.85 \mathrm{E}-08$ \\
\hline F11 & -2.0626 & $1.36 \mathrm{E}-15$ & -2.0626 & $1.36 \mathrm{E}-15$ & -2.06261 & 3.30E-09 \\
\hline F12 & -1 & 0 & -1 & 0 & -1 & 0 \\
\hline F13 & $-5.19 E-01$ & $1.92 \mathrm{E}-01$ & -0.6709 & 4.17E-03 & $-6.74 \mathrm{E}-01$ & $1.13 \mathrm{E}-16$ \\
\hline F14 & -50 & 0 & -50 & 0 & $-5.00 E+01$ & $8.03 \mathrm{E}-05$ \\
\hline F15 & -7.70E-01 & $1.46 \mathrm{E}-01$ & -0.869 & $1.13 \mathrm{E}-16$ & $-8.69 \mathrm{E}-01$ & $1.13 \mathrm{E}-16$ \\
\hline F16 & $-1.89 E+01$ & $\begin{array}{c}1.77166 \\
3\end{array}$ & -19.1796 & 4.25E-02 & $-1.92 \mathrm{E}+01$ & 8.18E-06 \\
\hline F17 & -1.8013 & $6.78 \mathrm{E}-16$ & -1.8013 & $6.78 \mathrm{E}-16$ & -1.8013 & $6.78 \mathrm{E}-16$ \\
\hline F18 & 7.46E-01 & $1.24 \mathrm{E}-01$ & 3.713554 & 1.8568 & $3.38 \mathrm{E}-03$ & 7.16E-03 \\
\hline
\end{tabular}




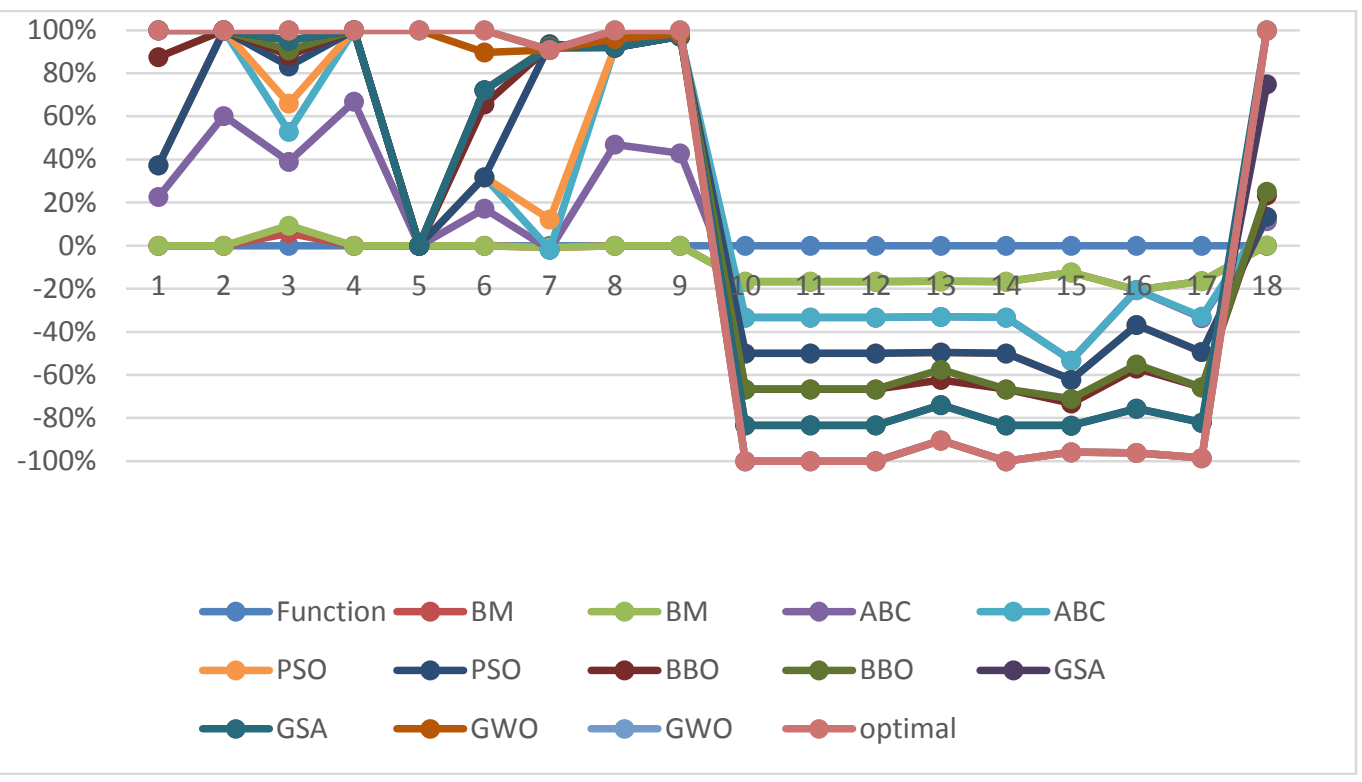

Fig.1. Unimodal benchmark function for BM with other algorithms

Table 4. Results of multimodal benchmark functions

\begin{tabular}{|c|c|c|c|c|c|c|}
\hline \multirow{2}{*}{ Function } & \multicolumn{2}{|c|}{ BM } & \multicolumn{2}{c}{ ABC } & \multicolumn{2}{c|}{ SSO } \\
\hline F19 & Average & Std & Average & Std & Average & Std \\
\hline F20 & -200 & 0 & -199.9998 & $1.02 \mathrm{E}-04$ & -200 & 0 \\
\hline F21 & 0 & 0 & $1.42 \mathrm{E}-05$ & $1.41 \mathrm{E}-05$ & $1.67 \mathrm{E}-16$ & $5.89 \mathrm{E}-16$ \\
\hline F22 & 0 & 0 & $4.52 \mathrm{E}-07$ & $5.79 \mathrm{E}-07$ & $6.97 \mathrm{E}-30$ & $2.48 \mathrm{E}-29$ \\
\hline F23 & $9.85874 \mathrm{E}-95$ & $2.88074 \mathrm{E}-94$ & $1.84 \mathrm{E}-09$ & $1.70 \mathrm{E}-09$ & $4.08 \mathrm{E}-104$ & $2.08 \mathrm{E}-103$ \\
\hline F24 & $3.6978 \mathrm{E}-32$ & $5.47382 \mathrm{E}-48$ & $2.20 \mathrm{E}-06$ & $2.14 \mathrm{E}-06$ & $8.52 \mathrm{E}-02$ & $4.08 \mathrm{E}-01$ \\
\hline F25 & -1 & 0 & -0.9999413 & $3.82 \mathrm{E}-05$ & -1 & 0 \\
\hline F26 & $1.00289 \mathrm{E}-89$ & $2.97672 \mathrm{E}-89$ & $1.33 \mathrm{E}-07$ & $1.36 \mathrm{E}-07$ & $6.77 \mathrm{E}-66$ & $3.71 \mathrm{E}-65$ \\
\hline F27 & 0 & 0 & $2.95 \mathrm{E}-31$ & $9.24 \mathrm{E}-31$ & 0 & 0 \\
\hline F28 & 0 & 0 & $5.26 \mathrm{E}-09$ & $7.66 \mathrm{E}-09$ & $6.96 \mathrm{E}-16$ & $2.23 \mathrm{E}-15$ \\
\hline F29 & 0.00156 & $2.1684 \mathrm{E}-19$ & $1.72 \mathrm{E}-03$ & $1.44 \mathrm{E}-04$ & $1.51 \mathrm{E}-02$ & $6.98 \mathrm{E}-02$ \\
\hline F30 & 0 & 0 & $1.06 \mathrm{E}-05$ & $8.71 \mathrm{E}-06$ & $1.33 \mathrm{E}-16$ & $5.70 \mathrm{E}-16$ \\
\hline F31 & $7.10209 \mathrm{E}-05$ & $4.18697 \mathrm{E}-05$ & $1.36 \mathrm{E}-04$ & $8.18 \mathrm{E}-05$ & $2.66 \mathrm{E}-02$ & $8.52 \mathrm{E}-02$ \\
\hline F32 & 0.002583809 & 0.002412803 & $2.04 \mathrm{E}-02$ & $1.08 \mathrm{E}-02$ & $1.86 \mathrm{E}+03$ & $6.52 \mathrm{E}+03$ \\
\hline & & & & & & \\
\hline
\end{tabular}


PEN Vol. 7, No. 3, September 2019, pp.1054-1066

\begin{tabular}{|c|c|c|c|c|c|c|}
\hline $\mathbf{F 3 3}$ & 0.014140107 & 0.00435564 & $8.93 \mathrm{E}-03$ & $2.97 \mathrm{E}-03$ & $6.95 \mathrm{E}-01$ & $7.72 \mathrm{E}-01$ \\
\hline $\mathbf{F 3 4}$ & 0.3979 & 0 & 0.39789 & $1.69 \mathrm{E}-16$ & $4.82 \mathrm{E}-01$ & $4.63 \mathrm{E}-01$ \\
\hline $\mathbf{F 3 5}$ & 3 & 0 & 3 & 0 & 3 & 0 \\
\hline $\mathbf{F 3 6}$ & -3.8628 & 0 & -3.8628 & $3.16 \mathrm{E}-15$ & -3.86278 & $1.36 \mathrm{E}-15$ \\
\hline $\mathbf{F 3 7}$ & -3.3224 & $1.33965 \mathrm{E}-15$ & -3.3224 & $1.36 \mathrm{E}-15$ & -3.27048 & $5.99 \mathrm{E}-02$ \\
\hline $\mathbf{F 3 8}$ & -6111.09 & 1029.511229 & $-1.20 \mathrm{E}+119$ & $6.05 \mathrm{E}+119$ & $-3.26 \mathrm{E}+03$ & $3.25 \mathrm{E}+03$ \\
\hline $\mathbf{F 3 9}$ & $2.05416 \mathrm{E}-93$ & $6.06452 \mathrm{E}-93$ & $2.50 \mathrm{E}-09$ & $2.06 \mathrm{E}-09$ & $2.27 \mathrm{E}-100$ & $8.67 \mathrm{E}-100$ \\
\hline $\mathbf{F 4 0}$ & 0 & 0 & $3.18 \mathrm{E}-05$ & $2.96 \mathrm{E}-05$ & $2.96 \mathrm{E}-17$ & $5.78 \mathrm{E}-17$ \\
\hline $\mathbf{F 4 1}$ & -106.7645 & 0 & -106.7645 & $7.23 \mathrm{E}-14$ & -103.115 & $2.00 \mathrm{E}+01$ \\
\hline $\mathbf{F 4 2}$ & 0.000025455 & 0 & $-1.97 \mathrm{E}+113$ & $1.03 \mathrm{E}+114$ & $7.12 \mathrm{E}+02$ & $4.47 \mathrm{E}+02$ \\
\hline $\mathbf{F 4 3}$ & -952.477 & 7.007255 & $-6 \mathrm{E}+111$ & $2.3 \mathrm{E}+112$ & -955.424 & 376.9031 \\
\hline
\end{tabular}

Table 4. Results of multimodal benchmark functions

\begin{tabular}{|c|c|c|c|c|c|c|}
\hline \multirow[t]{2}{*}{ unction } & \multicolumn{2}{|c|}{ BBO } & \multicolumn{2}{|c|}{ GSA } & \multicolumn{2}{|c|}{ GWO } \\
\hline & Average & Std & Average & Std & Average & Std \\
\hline F19 & 5.84E-09 & $1.15 \mathrm{E}-08$ & $9.31 \mathrm{E}-11$ & $5.16 \mathrm{E}-11$ & $8.74 \mathrm{E}-216$ & 0 \\
\hline F20 & -200 & 0 & -200 & 0 & -200 & 0 \\
\hline F21 & $3.02 \mathrm{E}-12$ & $1.62 \mathrm{E}-11$ & 0 & 0 & 0 & 0 \\
\hline F22 & 8.95E-10 & $2.23 \mathrm{E}-09$ & $1.85 \mathrm{E}-20$ & $1.43 \mathrm{E}-20$ & $1.54 \mathrm{E}-07$ & $1.11 \mathrm{E}-07$ \\
\hline F23 & $7.83 \mathrm{E}-16$ & $2.00 \mathrm{E}-15$ & $3.11 \mathrm{E}-20$ & 4.09E-20 & 0 & 0 \\
\hline F24 & $9.32 \mathrm{E}-09$ & $1.43 \mathrm{E}-08$ & $7.21 \mathrm{E}-20$ & $7.49 \mathrm{E}-20$ & $3.46 \mathrm{E}-08$ & $3.12 \mathrm{E}-08$ \\
\hline F25 & $-1.00 E+00$ & $1.21 \mathrm{E}-05$ & -1 & 0 & -1 & 0 \\
\hline F26 & $2.80 \mathrm{E}-08$ & $5.51 \mathrm{E}-08$ & 1.57E-21 & $1.19 \mathrm{E}-21$ & $8.42 E-204$ & 0 \\
\hline F27 & $2.26 \mathrm{E}-69$ & $1.24 \mathrm{E}-68$ & $1.72 \mathrm{E}-99$ & $4.23 E-99$ & 0 & 0 \\
\hline F28 & $1.66 \mathrm{E}-13$ & $9.09 \mathrm{E}-13$ & $5.33 \mathrm{E}-03$ & $6.96 \mathrm{E}-03$ & 0 & 0 \\
\hline F29 & $1.89 \mathrm{E}-03$ & $4.76 \mathrm{E}-04$ & $3.42 \mathrm{E}-03$ & $2.05 \mathrm{E}-03$ & $1.57 \mathrm{E}-03$ & $2.40 \mathrm{E}-07$ \\
\hline F30 & $4.39 \mathrm{E}-15$ & $2.35 \mathrm{E}-14$ & $2.02 \mathrm{E}-02$ & $3.20 \mathrm{E}-02$ & 0 & 0 \\
\hline F31 & $1.99 \mathrm{E}-04$ & $1.05 \mathrm{E}-04$ & $7.38 \mathrm{E}-05$ & 5.67E-05 & 4.95E-07 & $6.10 \mathrm{E}-07$ \\
\hline F32 & $1.80 \mathrm{E}-02$ & $1.57 \mathrm{E}-02$ & $2.84 \mathrm{E}-02$ & $3.32 \mathrm{E}-02$ & 1.07E-01 & 2.61E-01 \\
\hline F33 & $1.44 \mathrm{E}-03$ & $9.17 E-04$ & 4.47E-03 & $2.06 \mathrm{E}-03$ & $9.72 \mathrm{E}-04$ & $9.03 E-04$ \\
\hline F34 & $3.98 \mathrm{E}-01$ & $1.69 \mathrm{E}-16$ & 0.3979 & 0 & $3.98 \mathrm{E}-01$ & $5.98 \mathrm{E}-07$ \\
\hline
\end{tabular}


PEN Vol. 7, No. 3, September 2019, pp.1054-1066

\begin{tabular}{|c|c|c|c|c|c|c|}
\hline F35 & 3 & 0 & 3 & 0 & 3 & $9.28 \mathrm{E}-06$ \\
\hline $\mathbf{F 3 6}$ & -3.8628 & $3.16 \mathrm{E}-15$ & -3.8628 & $1.83 \mathrm{E}-05$ & -3.86208 & $1.94 \mathrm{E}-03$ \\
\hline $\mathbf{F 3 7}$ & -3.27472 & $5.94 \mathrm{E}-02$ & -3.3224 & $1.36 \mathrm{E}-15$ & -3.26425 & $9.99 \mathrm{E}-02$ \\
\hline $\mathbf{F 3 8}$ & $-8.20 \mathrm{E}+03$ & $5.72 \mathrm{E}+02$ & $-2.84 \mathrm{E}+03$ & $4.00 \mathrm{E}+02$ & $-6.10 \mathrm{E}+03$ & $7.90 \mathrm{E}+02$ \\
\hline F39 & $3.98 \mathrm{E}-02$ & $1.03 \mathrm{E}-01$ & $6.25 \mathrm{E}-21$ & $7.71 \mathrm{E}-21$ & 0 & 0 \\
\hline F40 & $6.55 \mathrm{E}-02$ & $1.02 \mathrm{E}-01$ & 0 & 0 & 0 & 0 \\
\hline F41 & $-1.06 \mathrm{E}+02$ & 3.551725 & -106.765 & $7.23 \mathrm{E}-14$ & $-1.06 \mathrm{E}+02$ & 3.551734 \\
\hline F42 & $4.67 \mathrm{E}+01$ & $6.48 \mathrm{E}+01$ & 206.868 & $8.14 \mathrm{E}+01$ & $5.92 \mathrm{E}+01$ & $7.46 \mathrm{E}+01$ \\
\hline F43 & -791.281 & 147.3338 & -727.028 & 123.868 & -922.428 & 208.9099 \\
\hline
\end{tabular}

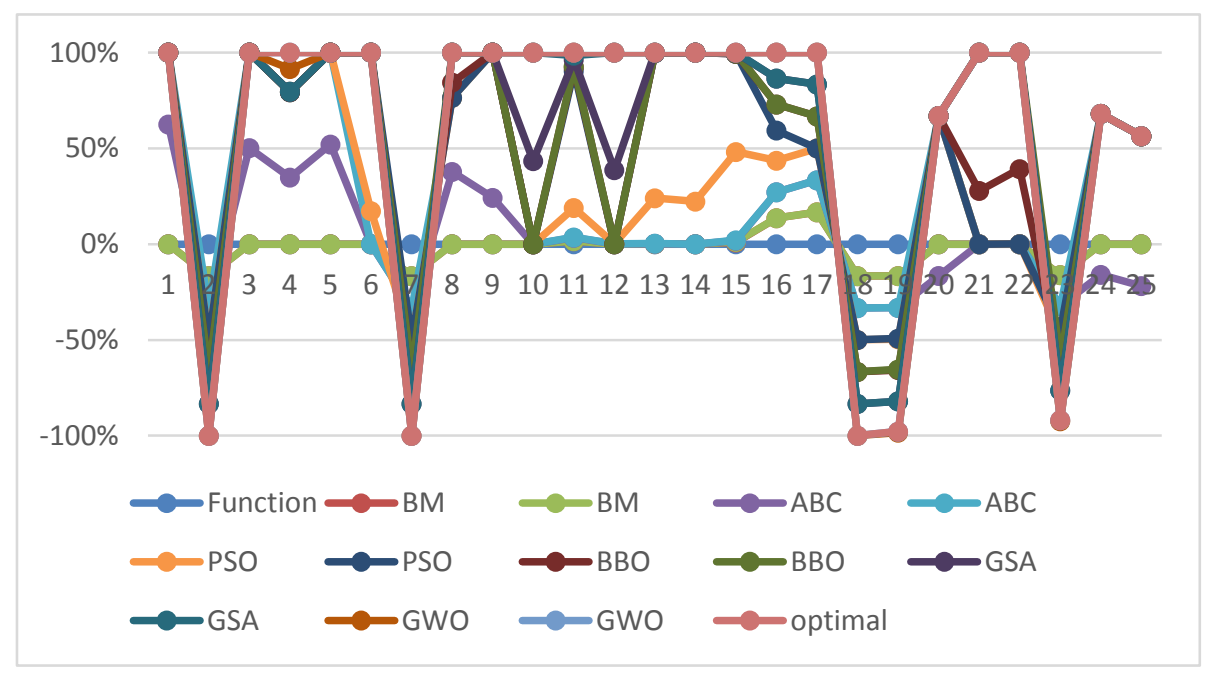

Fig.2. Multimodal benchmark function for BM with other algorithms

According to the results in table 3, BM algorithm achieved the optimal value in eight test functions (F5, F6, F7, F10, F12, F14, F15 and F16), while it is converging to the optimum values in nine test functions (which are F1, F2, F3, F4, F8, F9, F11, F13 and F17); it is worth to mention that those values are better compared to the values of the other selected algorithms using the same test functions. Also in the remaining test function (F18); BM is able to be near the best values of the selected algorithms.

The results BM show that it is very competitive and efficient compared to the algorithms mentioned above. BM was found to be superior to all other algorithms in most tests. Those results demonstrated the effectiveness, flexibility and accuracy of the presented algorithm.

BM was tested on 25 multi-model test functions, at which BM is better and more efficient than the other algorithm; also, BM can control the exploitation better than other algorithms.

Based on the results that have been listed in table 4, the BM algorithm achieved the optimal value in 14 test functions (F23, F24, F25, F26, F27, F32, F33, F34, F35, F36, F37, F38, F40, F41 and F42), while it is very close to the optimal values in 11 test functions (F19, F20, F29, F30, F31, F35 and F49); it is worth to mention that those values are better compared the values of the other selected algorithms using the same test functions. Also in the remaining four test functions (F21, F22, F28 and F43), BM is able to be near the best values of the selected algorithms.

The obtained results show that BM algorithm achieved better results in finding optimal solution value in the multi- model function, compared to the results of the unimodal. This reflecting the superiority of BM in the exploration search. 


\section{Conclusion and future work}

The presented work introduced a novel algorithm of swarm optimization based on the social behaviour of Blue Monkey; BM was suggested as a substitute technique to solve problems of optimization. In the suggested $\mathrm{BM}$ algorithm, the solutions were necessary to upgrade their positions according to the position of the optimal solution that has been achieved so far. The position updating enables the solutions to move towards or outwards the destination point for guaranteeing the exploitation and exploration of the search space. Fortythree test functions have been utilized for testing the power and efficiency of BM in terms of exploitation and exploration. The achieved results demonstrated that BM has the ability exceed ABC, GSA, PSO, GWO and BBO. The results that are obtained from the unimodal test functions showed the exploitation superiority of BM algorithm. After that, BM exploration ability was demonstrated by the results that are obtained from the multimodal benchmark functions. The next work is to build a multi-objective kind of the BM algorithm.

\section{References}

[1] E. Bonabeau, M. Dorigo, and G. Theraulaz, "Swarm Intelligence: From Natural to Artificial Systems," Oxford Univ. Press Usa, 1999.

[2] M. Dorigo, M. Birattari, and T. Stutzle, "Ant Colony Optimization," Comput. Intell. Soc”. IEEE. 2006, Vol. 1, Pp. 28-39.

[3] A. Kaveh, M. Khayatazad. “A New Meta-Heuristic Method: Ray Optimization”. Computers \& Structures 112-113:283-94.,2012.

[4] K. Ahmed, B. Al-Khateeb and M. Mahmood, "Application of Chaos Discrete Particle Swarm Optimization Algorithm On Pavement Maintenance Scheduling Problem," Cluster Computing, Https://Doi.Org/10.1007/S10586-018-2239-3.,2018.

[5] B. Horvat, BK. Berges, P. Lusso," Recent Developments in Animal Models for Human Herpesvirus 6a and 6b," Curr Opin Virol.; 9: 97-103. Doi: 10.1016/J.Coviro.2014.09.012 Pmid: $25462440 ., 2014$.

[6] G. B. Wang. "Swarm Intelligence in Cellular Robotic Systems," Goldberg, David. 1989. "Genetic Algorithms in Optimization, Search and Machine Learning., 1993.

[7] Jr. Koza, "Genetic Programming as A Means for Programming Computers by Natural Selection", Stat. Computer., Vol. 4, No. 2, Pp. 87-112, 1994.

[8] L. Lin and M. Gen, "Auto-Tuning Strategy for Evolutionary Algorithms: Balancing Between Exploration and Exploitation”, Soft Compute., Vol. 13, No. 2, Pp. 157-168, 2009.

[9] H. Du, X, Wu, J. Zhuang, "Small-World Optimization Algorithm for Function Optimization". Advances in Natural Computation, 264-73.,2006.

[10] D. Karaboga and B. Basturk, "A Powerful and Efficient Algorithm for Numerical Function Optimization: Artificial Bee Colony (ABC) Algorithm”, J. Glob. Optim., Vol. 39, No. 3, Pp. 459471, 2007.

[11] H. Shah-Hosseini, "Principal Components Analysis by The Galaxy-Based Search Algorithm: A Novel Metaheuristic for Continuous Optimisation,”, Int. J. Comput. Sci. Eng., Vol. 6, Pp. 132-140, 2011.

[12] B. Basturk and.D. Karaboga, "An Artificial Bee Colony (ABC) Algorithm for Numeric Function Optimization,”. IEEE Swarm Intelligence Symposium 4-12.,2006.

[13] K. D. Basturk, "An Artificial Bee Colony (ABC) Algorithm for Numeric Function Optimization," IEEE Swarm Intell. Symp., Pp. 4-12, 2006.

[14] D. Goldberg, "Genetic Algorithms in Optimization, Search and Machine Learning," Addison Wesley, New York. Eiben Ae, Smith Je, Ed. 2003 Introd. to Evol. Comput. Springer. Jacq J, Roux 
C Regist. Nonsegmented Images Using a Genet. Algorithm. Lect. Notes Comput. Sci., Vol. 905, Pp. 205-11, 1989.

[15] Z. Kifele, G. Belay G, A. Bekele, "Population Size, Group Composition and Behavioral Ecology of Geladas (The Ropithec Us Gelada) And Human-Gelada Conflict in Wonchit Valley," Ethiopia. J Biol Sci 16: 1248-1259.,2013.

[16] K. Abie, A. Bekele A "Population Estimate, Group Size and Age Structure of the Gelada Baboon (The Ropithecus Gelada) Around Debre-Libanos," Northwest Shewa Zone, Ethiopia. Glob J Sci Fr R Biol Sci 17: 27-33.,2017.

[17] C. Chapman and L. Chapman "Constraints On Group Size in Red Colobus and Red-Tailed Guenons: Examining The Generality of the Ecological Constraints Model,” Int J Primatol 21: 565585.2002.

[18] E. Kibebew and K. Abie "Population Status, Group Size, And Threat to Boutourlini's Blue Monkeys (Cercopithecus Mitis Boutourlinii) In Jibat Forest," Ethiopia. J Ecosyst Ecography 7: 230. Doi:10.4172/2157-7625.1000230.,2017.

[19] Sg. Solomon and Mg. Rosa, "A Simpler Primate Brain: The Visual System of the Marmoset Monkey," Front Neural Circuits. 2014; 8: 1-24. Doi: 10.3389/Fncir.2014.00096.

[20] S. Mirjalili, A. H. Gandomi, S. Z. Mirjalili, S. Saremi, H. Faris, and S. M. Mirjalili, "Salp Swarm Algorithm: A Bio-Inspired Optimizer for Engineering Design Problems," Adv. Eng. Softw., Vol. 114, Pp. 163-191, 2017.

[21] J. Kennedy and R. Eberhart R. "Particle Swarm Optimization, In Neural Networks." IEEE. International Conference 1942-48.,1995.

[22] E. Rashedi, N. Hossein, and S. Saeid Saryazdi. "Gsa: A Gravitational Search Algorithm," Information Sciences 179(13):2232-48. ,2009.

[23] S. Mirjalili, M.M. Seyed, and L. rew, "Grey Wolf Optimizer," Advances in Engineering Software 69:46-61.,2014.

[24] Benjamin Duraković, Hazim Bašić, "Continuous Quality Improvement in Textile Processing by Statistical Process Control Tools: A Case Study of Medium-Sized Company", Periodicals of Engineering and Natural Sciences, Vol. 1 No. 1(2013), ISSN 2303-4521, pp 39-46.,2013. 